\title{
Formación de docentes para la creación de cursos virtuales en la enseñanza del español como segunda lengua
}

Por: Magíster María Gabriela Amador Solano¹, Instituto Tecnológico de Costa Rica, y Máster Julia Espinoza-Guzmán², Instituto Tecnológico de Costa Rica

Recibido: $\quad 20$ de marzo de 2017

Aprobado: 29 de mayo de 2017

\section{Resumen}

Con el fin de aprovechar el potencial ofrecido por las TIC en la educación, es necesario crear propuestas de cursos virtuales que permitan ampliar la oferta educativa en las instituciones de educación superior, lo cual representa una ventaja competitiva para las universidades. De esta idea, se diseñó la investigación sobre un programa para formar profesores en la creación de cursos virtuales, en el área específica de enseñanza del Español como Segunda Lengua. La investigación es cualitativa. El objetivo es proponer un plan de formación docente para la creación de cursos virtuales, en la enseñanza del español como segunda lengua, a partir de criterios pedagógicos establecidos por expertos. Para ello, se tomaron en cuenta los siguientes criterios: el diseño instruccional basado en el contexto y en la necesidad de capacitación, las estrategias de aprendizaje y evaluación, la creación de rúbricas y la confección de material didáctico. El estudio se llevó a cabo con docentes del programa Técnico en la Enseñanza del Español como Segunda Lengua, del Instituto Tecnológico de Costa Rica, quienes previo a la capacitación, no tenían ninguna formación virtual. Entre los resultados de este proyecto, cada docente logró crear su diseño para un curso virtual a futuro, a partir de los criterios anteriormente mencionados.

\begin{abstract}
Education of teachers in order to create virtual courses for Spanish as a Second language

In order to take advantage of the potential of ITs in education, it is necessary to create proposals of virtual courses which allow a wider variety of educational opportunities in higher education institutions, which is advantageous for universities. Departing from this idea, an investigation project was designed regarding a program to prepare teachers for the creation of virtual courses, specifically for Spanish as a Second Language. The research is qualitative. The objective is to propose a plan to prepare teachers in the creation of virtual courses for Spanish as a Second Language departing from the pedagogical principles posed by experts. In order to do so, the following criteria were taken into account: the instructional design based on context and the need for training, the learning strategies and evaluation, the creation of rubrics and the design of teaching materials. The research was carried out by teachers of an undergraduate program of Teaching of Spanish as a Second Language at Costa Rica Institute of technology, who prior to the research had no or very little background on virtual courses. Among the results of the study, each teacher was able to create the design of a potential virtual course, based on the criteria mentioned above.
\end{abstract}

1 María Gabriela Amador se desempeña como docente e investigadora de la Escuela Ciencias del Lenguaje, del Instituto Tecnológico de Costa Rica. Es graduada de Magíster en Español como Segunda Lengua, de la Universidad de Costa Rica y actualmente es estudiante del Doctorado en Filosofía y Letras con énfasis en Lingüística Aplicada a la Enseñanza de Lenguas, de la Universidad de Alicante, España. Contacto: gamador@itcr.ac.cr

2 Julia Espinoza labora como profesora e investigadora de las carreras de Ingeniería en Computación y Administración de Tecnologías de Información, en el Instituto Tecnológico de Costa Rica. Su formación incluye una maestría en Tecnología Educativa con énfasis en procesos (Universidad de Estudios Superiores de Monterrey, México) otra maestría en Diseño, Gestión y Dirección de Proyectos (Universidad Politécnica de Cataluña, España), una licenciatura en Ciencias de la Educación con Énfasis en Docencia (Universidad Estatal a Distancia, Costa Rica) y una Ingeniería en Computación Administrativa (Instituto Tecnológico de Costa Rica). Contacto: juliaespinoza@itcr.ac.cr.
María Gabriela Amador Solano y Julia Espinoza-Guzmán. Formación de docentes para la creación de cursos virtuales en la enseñanza del español como segunda lengua. Revista Comunicación. Año 38, volumen 26, número 1, enero - junio, 2017. Instituto Tecnológico de Costa Rica. ISSN: 0379-3974 / e-ISSN: 1659-3820. 


\section{INTRODUCCIÓN}

Vivimos en una era de enormes posibilidades para incluir las Tecnologías de la Información y Comunicación (TIC) en el ámbito educativo. Esa realidad digital en que estamos inmersos es un reto para la institución, el docente y para el alumno. Con el fin de aprovechar el potencial ofrecido por las TIC en la educación, es necesario plantear propuestas de cursos virtuales que permitan ampliar la oferta educativa en las instituciones de educación superior, lo cual es una ventaja competitiva para las universidades (Savic, Stankovic y Janackovic, 2012).

El tema de cursos virtuales cobra importancia en el contexto de las universidades costarricenses, ya que la Oficina de Planificación de la Educación Superior en el Plan Nacional de la Educación Superior Universitaria Estatal 2011-2015 (CONARE, 2011) especifica un conjunto de ejes, objetivos y acciones acerca del uso de las TIC en relación con las modalidades de enseñanza universitaria a distancia, virtual y bimodal. Dicho Plan propone una creciente necesidad de investigación en el campo de la educación virtual que permita darle sostenibilidad y credibilidad a su implementación. Por su parte, el Instituto Tecnológico de Costa Rica (ITCR) en su Plan Estratégico 2011-2015, establece en uno de los objetivos estratégicos "Fortalecer la incorporación de las tecnologías de información y comunicación en el mejoramiento del quehacer académico y de apoyo a la academia." (2012, p. 14).

Para cumplir con estos propósitos, es evidente la necesidad de capacitar a los docentes para la inclusión de actividades con el uso de la tecnología en sus planes didácticos, cursos bimodales, programas virtuales o a distancia. Asimismo, se trata de que el docente comprenda la diferencia que existe al dar seguimiento virtual a cada uno de sus estudiantes para verificar las habilidades que van adquiriendo lo cual es muy distinto a dejar material "colgado" en la plataforma para que los alumnos lo lean cuando deseen, sin ninguna supervisión del caso.

Por tal motivo, para asegurarse una formación de calidad en el diseño de programas de formación para docentes es necesario aplicar criterios pedagógicos que incluyan la tecnología educativa en el diseño de cursos virtuales para la enseñanza de una segunda lengua. En este contexto se realizó la in- vestigación. Se crearon diversos módulos para que el educador fuera aprendiendo a realizar su propia propuesta virtual, a partir de un diseño instruccional basado en el contexto y en la necesidad de capacitación, la creación de rúbricas, la confección de material didáctico y el perfil de salida del público meta.

\section{NECESIDAD DE CURSOS VIRTUALES DE CALIDAD}

A nivel mundial se han desarrollado varias propuestas con respecto a los criterios de calidad de cursos virtuales. De hecho, en la literatura se proponen factores críticos que inciden en la calidad de un curso que utilice las TIC. Estos factores pueden ser visualizados desde diversas perspectivas, por ejemplo, desde el punto de vista de los actores involucrados, de las acciones pedagógicas, de los contenidos, de los procesos y acciones institucionales o de la plataforma tecnológica (Área, San Nicolás y Fariña, 2010; Cabero, 2006; Clark y James, 2012; García-Aretio, 2009; González y Farnóz, 2009; Imbernón, Silva y Guzmán, 2011; Ortega, 2007; Ortega y García, 2002; Sae-Khow, 2014; Salinas, Pérez y De Benito, 2008; Sarsa y Soler, 2012).

Un caso concreto, es la tesis presentada por Rico (2012) que plantea la revisión sistemática y continua de los procesos de enseñanza-aprendizaje virtual para generar una propuesta de modelo tomando como base criterios de estándares internacionales. En este proyecto se concluye que "no se puede determinar que exista una única aproximación pedagógica, tecnológica u organizativa al e-learning. Existen muchas y diversas. Lo auténticamente relevante será determinar cuáles son los elementos que definen la elección de un modelo u otro y cómo, a partir de ahí, se establece la coherencia entre los diferentes elementos" (Rico, 2012, p. 108).

Por otro lado, Pernalete, Cánchica y Coello (2010) en su investigación analizan una comparación de estándares y modelos de calidad asociados a ambientes de enseñanza-aprendizaje para el e-learning. Concluyen sobre la importancia de generar un diseño de curso que se pueda adaptar a un contexto real y a las necesidades de los participantes. Recomiendan, además, el uso de marcos de aplicación en un plan piloto para facilitar a los centros de 


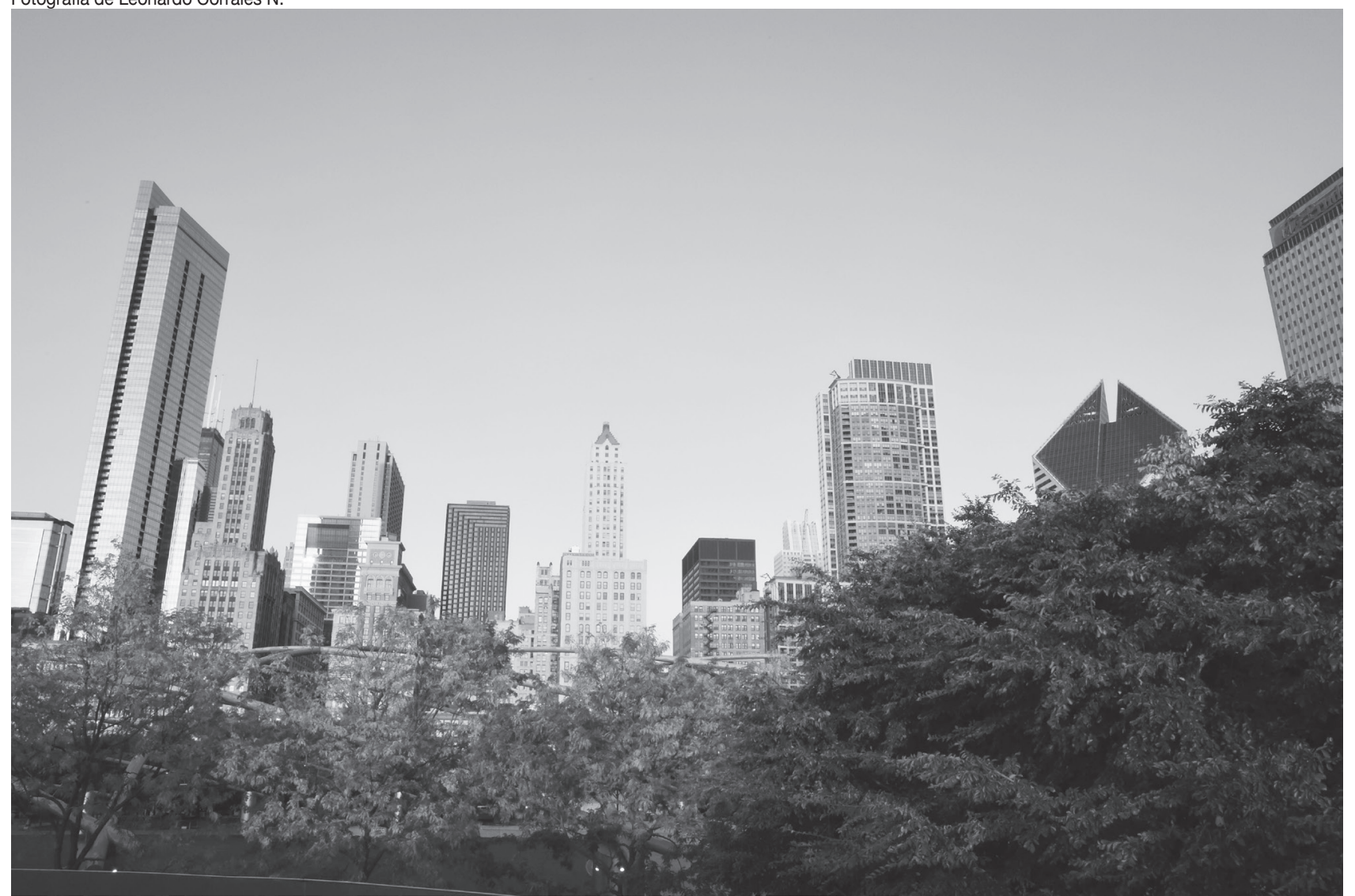

enseñanza, la selección y adopción de estándares, con el fin de regular las normas de diseño para un programa virtual. En este sentido, se justifica la importancia de realizar una capacitación previa a la oferta educativa, para poder retroalimentar el proceso y verificar resultados con respecto a los objetivos planteados.

Por su parte, Savic, Stankovic y Janackovic (2012) presentan un modelo híbrido para la evaluación de la calidad del e-learning que examina características, estándares, criterios, e indicadores de calidad. Ejarque, Buendía y Hervás (2008), por su parte, exponen un método que permite evaluar en forma sistemática y estructurada las experiencias de enseñanza y aprendizaje apoyadas en plataformas e-learning en el marco del Espacio Europeo de Educación Superior.

En cuanto a las aulas virtuales o portales del curso, los expertos identifican, también aspectos que contribuyen a mejorar la calidad del curso. Para Área, San Nicolás y Fariña (2010) es fundamental que las aulas virtuales superen el modelo reduccionista de "colgar" apuntes del curso. Más bien deben procurar que el estudiante reciba el contenido informativo en diferentes formatos, se ofrezca un conjunto variado de actividades, los procesos de comunicación sean fluidos y constantes. La puesta en práctica del plan piloto de esta investigación presentada ayuda a que los docentes que preparan sus cursos, sean al mismo tiempo estudiantes, con el fin de que analicen y puedan dar respuesta a las inquietudes surgidas durante el proceso.

Uno de los componentes esenciales de los cursos virtuales son las metodologías para aprender, que como indican Salinas, Pérez y De Benito (2008) deben tener como centro de atención el estudiante. Mientras que Sarsa y Soler (2012) hacen una amplia revisión de estudios y factores que han sido analizados para determinar la calidad del e-learning. Por otro lado, para Wang (2007) el nivel y tipo de interacción entre estudiantes y profesores juega un papel fundamental en la calidad del aprendizaje y del curso. 
Entre otras investigaciones consultadas sobre la puesta en práctica de modelos pedagógicos virtuales, está la de Rodríguez (2012), titulada "La satisfacción del usuario en la enseñanza virtual: El caso del sistema universitario andaluz". El objetivo planteado fue evaluar cuáles son los factores que influyen en la satisfacción de los estudiantes que cursan materias virtuales. Al respecto, se determinaron tres factores: diseño y la estructura del curso (planificación, materiales y evaluación); la funcionalidad de la plataforma tecnológica y la interacción con el profesor y los compañeros.

Alfonzo (2011), por su parte, determinó si las nociones de "autonomía" y "aprendizaje independiente", son o no complementarias con la interacción y el aprendizaje colaborativo. Al final del proyecto se consideró que la autonomía implica también interacción con los compañeros y profesores. Es preciso que haya interacción para poder hablar de aprendizaje significativo.

Por otro lado, Nieto (2013), planteó el diseño de un modelo en el cual se reflejara la cultura, intereses y limitaciones de la región Argentina, en donde se efectuó el análisis. Al respecto, se concluye que es necesario pensar y planificar modelos pedagógicos de enseñanza virtual adecuados a la realidad social de la región, para que los recursos de la tecnología educativa faciliten el acceso democrático a la educación.

En otro caso, Jiménez (2013), pretende dar a conocer el deber ser del profesor universitario y su responsabilidad como usuario y difusor de las nuevas tecnologías en el contexto educativo. Una de sus conclusiones es que la evaluación es más dinámica en el desarrollo de los cursos regulares, a través de la plataforma virtual, porque permite evaluar el aprendizaje de los estudiantes de manera más amena e innovadora. Este aspecto es fundamental para tomarlo en cuenta en el estudio, pues los profesores no contaban con experiencia $100 \%$ virtual antes de la investigación.

Finalmente, vale resaltar dos investigaciones realizadas en el ITCR en el ámbito de cursos bimodales. La primera, Solano (2010) logra comprobar que la implementación de estrategias propias de la virtualidad fomenta el desarrollo de las habilidades requeridas para la comunicación escrita.
Por su parte, la segunda, Rodríguez, Espinoza y Moreira (2014) estudiaron las diferencias de los aprendizajes de estudiantes de un curso de Cálculo y Álgebra Lineal de la Escuela de Matemática, del ITCR, en un grupo presencial y otro bimodal. Sobre esto concluyen que en ambos casos, el aprendizaje es significativo y rescatan la importancia del papel del docente al usar las herramientas tecnológicas y sobre el aprovechamiento potencial del educando al desarrollar las actividades en la plataforma TEC Digital $^{3}$.

\section{CRITERIOS PEDAGÓGICOS PARA OFRECER CURSOS VIRTUALES}

Se realizó una comparación de diversos estudios relacionados, para conocer los criterios pedagógicos destacados y reconocidos por los expertos como importantes en relación con el desarrollo de cursos virtuales. A partir de esta comparación se destacan los siguientes:

\section{Diseño instruccional.}

2. Estrategias de aprendizaje y de evaluación centradas en el estudiante.

3. Rúbricas de evaluación.

4. Materiales didácticos.

En primera instancia, se considera el diseño instruccional (DI) como elemento fundamental para tomar acciones tecnopedagógicas que ocurrirán en el espacio de aprendizaje. Para Arshavskiy (2014) el DI constituye una herramienta que guía la "estructura de cualquier curso y promueve tanto el aprendizaje significativo como el activo" (2014, p. 4). Por su parte, Belloch $(2013$, p. 2) cita a Richey, Fields y Foson (2001) para apuntar que el diseño instruccional "supone una planificación instruccional sistemática que incluye la valoración de necesidades, el desarrollo, la evaluación, la implementación y el mantenimiento de materiales y programas".

El diseño instruccional no es exclusivo para ambientes virtuales de aprendizaje. Sus orígenes se remontan a casi un siglo atrás (Arshavskiy, 2014). No obstante, el auge en las últimas décadas del aprendizaje en línea ha hecho que el DI cobre mayor importancia, dado que implica la planeación

3 TEC Digital es la plataforma oficial del Instituto Tecnológico de Costa Rica (ITCR) 
detallada de un ambiente de aprendizaje. Existe concordancia entre el planteamiento de Arshavskiy y lo que indica Fernández-Robles y Cabero, en el sentido de que "la planificación y el diseño de la instrucción son definitivas en todas las modalidades formativas, ya que es lo que permite pensar de manera coherente la secuencia de los aprendizajes." (2016, p. 5).

La primera fase del DI corresponde al diagnóstico del entorno educativo donde se impartirá el curso, el plan de estudios, así como de la población a la que está dirigida la formación: sus características etarias, formación previa, aspectos de aprendizaje por atender e infraestructura tecnológica existente (Gil, 2012). Posteriormente se desarrollan un conjunto de pasos que permiten establecer el contenido por aprender, los objetivos educativos, las actividades didácticas, así como los materiales por desarrollar y la evaluación o comprobación de los objetivos.

El segundo criterio por considerar en la propuesta es la relación entre estrategias de aprendizaje y de evaluación enfocadas en los estudiantes, así como técnicas asociadas y posibles herramientas para implementarlas. Al respecto, Salinas, Pérez y De Benito (2008), enfatizan la importancia de que las estrategias, técnicas y actividades estén diseñadas para los estudiantes, con el objetivo de sacar el mayor provecho de las redes de comunicación que ofrecen las TIC.

El tercer criterio de la propuesta es la elaboración y uso de rúbricas de evaluación. La elección de las rúbricas, según Fallas (2005) obedece a que "una de las mayores dificultades que enfrentan los facilitadores de los cursos en línea, es la necesidad de contar con herramientas que les permitan evaluar el desempeño del estudiante en forma objetiva y consistente $^{\prime \prime}($ p.1). Cada profesor participante en la formación, debió elegir un tema de su curso para crear este instrumento, con el fin de poder calificar de forma objetiva a sus educandos. El proceso de construcción de la rúbrica se basó en lo propuesto por Gatica-Lara y Uribarren-Berrueta (2013).

Como cuarto y último criterio de la propuesta, se plantea la creación de los materiales educativos asociados directamente al diseño instruccional de cada curso y que deben estar desarrollados en función de la población meta, de las actividades de aprendizaje propuestas en el diseño instruccional y además que cumplan con los lineamientos propuestos por el TEC Digital. De esa manera, "un material didáctico es aquel que ha sido específicamente diseñado para el aprendizaje, que contiene en sí mismo una concepción del aprendizaje y de aquello que los estudiantes tienen que hacer para aprender, sea esto explícito o no" (Odetti, 2013, p. 5).

\section{FORMACIÓN EN LA ENSEÑANZA DE UNA SEGUNDA LENGUA}

De acuerdo con las diversas investigaciones de la enseñanza de una segunda lengua, existe una estrecha relación entre la adquisición de idioma meta y el proceso conjunto del desarrollo de las cuatro habilidades comunicativas: expresión escrita, lectura, escucha y expresión oral. En este sentido, se toma en cuenta para la propuesta de capacitación docente el documento Ilamado Marco Común Europeo de Referencia para las Lenguas (MCER, Consejo de Europa, 2002).

El MCER es una guía para el profesor sobre el aprendizaje, enseñanza y evaluación de lenguas extranjeras. Busca orientar en el desarrollo de actividades comunicativas, por medio de conocimientos y destrezas, los cuales son el punto de partida de los niveles comunes de referencia para la adquisición de una lengua. "Describe de forma integradora lo que tienen que aprender a hacer los estudiantes con el fin de utilizar una lengua para comunicarse, así como los conocimientos y destrezas que tienen que desarrollar para poder actuar de manera eficaz". (Consejo de Europa, 2002, p.1).

Otra publicación recomendada a los docentes para este proyecto es el Plan Curricular del Instituto Cervantes (Instituto Cervantes, 2006). Resulta ser un tratamiento sistemático de los materiales necesarios para preparar los objetivos y los contenidos de los programas de enseñanza a nivel mundial. Los niveles propuestos en este documento son los que hace referencia el MCER (A1-A2, B1-B2, C1-C2).

El objetivo de incluir ambos textos en la capacitación se debe al énfasis del programa, centrado en la enseñanza de segundas lenguas. Por tanto, además de incluir el aprendizaje de herramientas tecnológicas en los diferentes módulos de la propuesta, 
también es fundamental el repaso de los estándares señalados por el sistema de referencia de lenguas, con el fin de que las actividades didácticas virtuales generadas por los participantes se planeen en función de lo que dicta el sistema de referencia de la enseñanza de un idioma.

Tal como afirma Pastor (2005) con el crecimiento del número de hablantes que quieren aprender un idioma, es lógico el aumento de la demanda de profesionalización de este tipo de docencia, lo cual genera una discusión de cómo debe prepararse y qué posibilidad de formación tiene el futuro profesor. Este argumento justifica en buena medida la necesidad de generar programas que formen a los docentes para desempeñarse con éxito en su labor.

\section{CARACTERÍSTICAS DEL ESTUDIO Y PROPUESTA METODOLÓGICA}

La investigación es de orden cualitativa pues se encarga de analizar la realidad del estudio a partir de la observación e interpretación de resultados. De hecho, la relación docente-educando implica un proceso que requiere de la interacción entre emisor y receptor, en consonancia con el currículo. En este sentido, en el grupo meta se aplicaron diferentes tipos de evaluación: diagnóstica, procesual y final. Estos instrumentos permitieron considerar los resultados arrojados al final de la investigación realizada.

La teoría sobre los tres tipos de evaluación que servirán para el análisis de datos, proviene de Juan Videla (sf), quien se refirió en su artículo titulado "Evaluación", a los siguientes criterios. Por un lado, la evaluación diagnóstica se realiza previo al comienzo del curso por impartir, con el fin de determinar estadísticas o datos que evidencien la necesidad de capacitación. Por otro lado, la evaluación procesual que "consiste en la valoración a través de la recogida continua y sistemática de datos (...) a lo largo del periodo de tiempo fijado para la consecución de unas metas u objetivos" (p.6). Por último, la evaluación final, la cual se refiere a la recolección de datos y su respectivo análisis, cuando se finaliza el tiempo previsto para la ejecución del programa académico.

Mediante el método de investigación se escogieron las siguientes estrategias cualitativas, las cuales

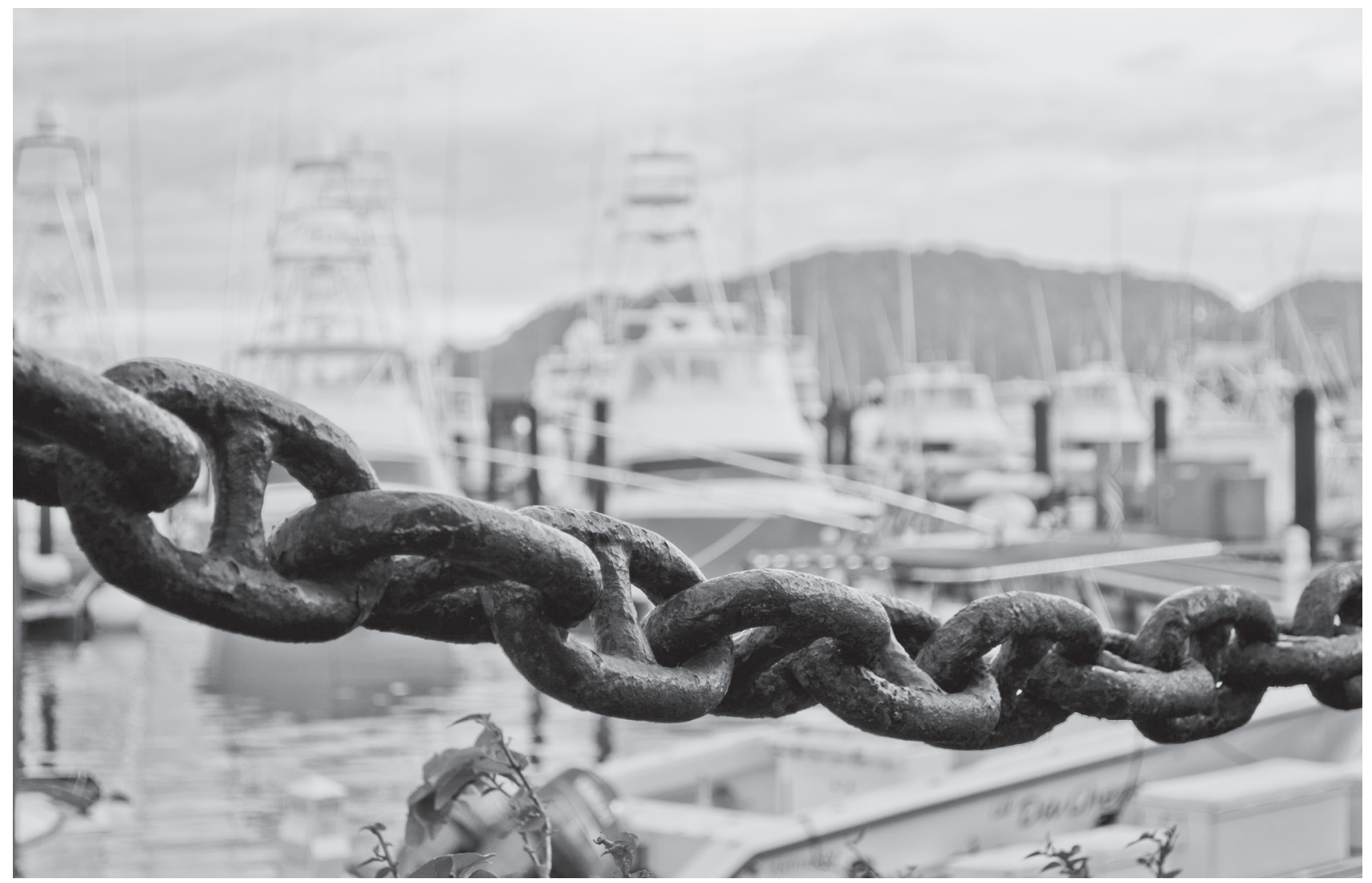

Fotografía de Leonardo Corrales N. 
fueron aplicadas a lo largo de todo el proceso: observación participante, entrevistas cualitativas y documentos oficiales de la institución respecto al uso de la plataforma y el tipo de capacitación docente.

En la observación, hay una interacción entre el investigador y el grupo meta, es decir, los profesores. En este sentido, las investigadoras fueron parte de la capacitación que recibieron los docentes, con el fin de tener una visión más detallada y profunda de los comportamientos que tienen respecto al rol como estudiantes. Se observó por medio de sistema de videoconferencia y a partir de los productos generados, cómo se sintieron emocionalmente en el proyecto, y si cumplió sus expectativas.

Con respecto a la entrevistas, se realizaron en varias etapas del estudio. Por ejemplo, en la etapa inicial, previo al curso, se realizó una entrevista a diez personas por cada uno de los 20 centros de enseñanza del español en Costa Rica. En la etapa procesual, la entrevista tuvo como objetivo verificar el perfil de ingreso de los participantes, pues eso dependería de las características profesionales, personales y técnicas. Mientras que en la etapa final, la entrevista se realizó a los docentes participantes para conocer sus impresiones sobre las clases, la metodología, las educadoras, la plataforma, la comunicación asertiva, el sistema de videoconferencia, entre otros aspectos.

Una vez aplicados los instrumentos, tomando en cuenta los datos recolectados y los documentos de la institución, el análisis de la información se realizó en tres fases: categorización, estructuración y teorización. Finalmente, se implementó la etapa de triangulación para obtener los resultados, en la que se interpretaron las informaciones desde diferentes ángulos, siguiendo los criterios que desencadenan los propósitos de evaluación planteados para el modelo formativo.

Por otro lado, es importante exponer cómo se hizo la selección de docentes para que recibieran la capacitación sobre la creación de cursos virtuales en el área afín. En total fueron cinco profesores, quienes corresponden al equipo docente del Programa Técnico en la Enseñanza del Español como Segunda Lengua (PT EESL), contratados de forma continua. Entre sus características por las cuales fueron escogidos están: experiencia laboral en dicho programa en mínimo cuatro promociones de estudiantes (2009-2016), resultados satisfactorios de 95 a 100 en evaluación docente, afinidad con el uso de herramientas tecnológicas, actitud positiva frente a la necesidad de capacitarse y preparación profesional en el campo. El PT EESL es el único programa a nivel Técnico (2017) en Costa Rica, por eso solo se trabajó con esta población.

El PT EESL, es una capacitación docente que prepara en las herramientas básicas requeridas para enseñar este idioma a extranjeros, de manera presencial en el aula. Nació en el año 2009, en la Escuela de Ciencias del Lenguaje, del Instituto Tecnológico de Costa Rica, a cargo de la Máster María Gabriela Amador Solano, especialista en esta área específica. Tiene una duración de un año intensivo, en total trece cursos: Medios digitales, Competencias tecnológicas, Niveles comunes de referencia, Creación de material didáctico, Turismo rural comunitario, Gramáticas A1-A2, B1-B2, C1-C2, Evaluación para los aprendizajes, Claves culturales, Métodos para la enseñanza, Fonética y tecnologías y Planeamiento didáctico.

Los profesores escogidos representan la mayoría del total de docentes, son cinco escogidos de siete que están contratados a medio tiempo. A pesar de tener dentro de sus características una afinidad por la tecnología, ninguno había tenido una experiencia $100 \%$ virtual de un programa completo de formación docente, previo a la capacitación. Este dato es muy interesante con respecto a los resultados.

Para desarrollar el programa de capacitación se utilizó la plataforma TEC Digital, propia y diseñada para el Instituto Tecnológico de Costa Rica, la cual dispone de herramientas para la comunicación sincrónica y asincrónica, el intercambio de recursos educativos en diferentes formatos, la gestión de las evaluación, gestión de rúbricas, entre otros servicios, lo que permite potenciar el aprendizaje más allá del espacio del aula física.

Se procedió a realizar una propuesta inicial de los criterios pedagógicos para la creación de cursos virtuales, a partir de la lectura y análisis de diversas investigaciones que plantean el tema. A partir de este estudio, se seleccionaron cuatro criterios, los cuales fueron mencionados anteriormente: 


\section{Imagen 1: Plan para la formación docente virtual, PT EESL}

\section{FORMACIÓN DOCENTE PARA AMBIENTES VIRTUALES}

Módulo introductorio
1 Semana
Módulo 1
Aprendizaje en línea,
en línea y combinado
1 Semana

Módulo 2

Web $2.0 y$

procesos educativos

1 Semana
- Descripción

Conoce la plataforma e incluye la información personal. Conoce las normas de netiqueta.

- Resultado

Navega fluidamente por la plataforma, localiza las herramientas, aplica las normas de netiqueta y actualiza su perfil académico en plataforma.

- Descripción

Conoce las diferencias entre aprendizaje presencial, en línea y combinado. Analiza roles que del estudiante y del docente, así como formas de interacción

- Resultado

Valora las opciones de aprendizaje presencial, en línea y combinado, justificando el contexto en que puede aplicar cada una, comparando roles de estudiantes y profesores, así como las formas de interacción y las herramientas tecnológicas para llevarlas a cabo

\section{- Descripción}

Considera la Web 2.0 y ayuda a repensar las posibilidades didácticas a la luz de las tecnologías de la información y la comunicación, así como desde el rol de profesores y estudiantes en este nuevo contexto

- Resultado

Panorama de la web 2.0 en el proceso de enseñanza-aprendizaje

Módulo 3

Proceso de diseño instruccional

1 Semana

- Descripción

Presenta el Diseño instruccional como proceso y se exploran herramientas para ambientes virtuales para la incorporación en el diseño instruccional del curso.

- Resultado

Conocimiento del proceso de diseño instruccional y herramientas tecnológicas.

\begin{tabular}{l|l|}
$\begin{array}{l}\text { Módulo 4 } \\
\text { Desarrollo del diseño }\end{array}$ & $\begin{array}{l}\text { Descripción } \\
\text { Cada participante aplica el proceso de diseño } \\
\text { instruccional } \\
\text { instruccional para generar la planificación detallada } \\
\text { del curso a su cargo en el PT EESL. }\end{array}$ \\
& $\begin{array}{l}\text { Resultado } \\
\text { La planificación didáctica del curso. }\end{array}$ \\
Módulo 5 & $\begin{array}{l}\text { Descripción } \\
\text { Materiales educativos }\end{array}$ \\
y rubricas & $\begin{array}{l}\text { profesor elabora rúbricas de evaluación y desarrollo } \\
\text { de materiales educativos. } \\
7 \text { Semanas }\end{array}$ \\
& $\begin{array}{l}\text { Resultado } \\
\text { Materiales educativos desarrollados y rúbricas }\end{array}$
\end{tabular}

Fuente: elaboración propia 
diseño instruccional, estrategias de aprendizaje y de evaluación centradas en el estudiante, técnicas asociadas y posibles herramientas para implementarlas, rúbricas de evaluación y creación de materiales educativos.

Basado en la propuesta teórica anterior, y en los recursos disponibles, se creó un plan para la formación docente virtual, compuesto por cinco módulos: el primero, Herramientas de la web 2.0; el segundo, Planificación didáctica para cursos virtuales; el tercero, Diseño Instruccional; el cuarto, Creación de rúbricas y el quinto, Creación de material didáctico para cursos e-learning. El plan de formación y el programa de cada módulo fueron diseñados por dos especialistas en e-learning, autoras de este artículo.

Cada una de las actividades didácticas se realizó a partir de la formación de docentes en la enseñanza de una segunda lengua, pues fue el requisito para poder participar en el plan piloto. Los interesados tuvieron la oportunidad de generar su propio material didáctico para las sesiones virtuales de un programa de enseñanza de lenguas. En este caso, todos los docentes trabajaron sus productos para el aprendizaje del idioma español. Cada uno de los educadores tuvo que participar como alumno, de los seis módulos (véase la imagen 1), uno introductorio y cinco temáticos, que abarcaron en la especificación realizada en la plataforma, sus respectivos objetivos, contenidos, actividades y evaluación.

Para el diseño de la formación de educadores, se emplearon diversas estrategias y técnicas para el aprendizaje y la evaluación, como por ejemplo aprendizaje significativo y aprendizaje colaborativo, las cuales fueron implementadas con herramientas como wiki, foro, y documentos en la nube, correo electrónico, redes sociales, herramientas para videollamadas y otras aplicaciones disponibles en la plataforma TEC Digital. En distintas actividades, a lo largo de la formación, se utilizaron esas funcionalidades tecnológicas y los profesores conocieron opciones de uso que les facilitaron la redacción del diseño instruccional.

En primer lugar, el módulo introductorio ofrece un preámbulo de todo el programa y de la plataforma virtual. En segundo lugar, en el módulo uno se determinan las diferencias entre aprendizaje presencial, en línea y combinado. Así, también se analizan los roles del docente y educando. El módulo dos tuvo como objetivo el conocimiento y aplicación de las herramientas web 2.0. Para el siguiente, el módulo tres, se hace la propuesta de un diseño instruccional para un curso virtual que desea generar cada participante. En este sentido, el módulo cuatro, sugiere aplicar el diseño realizado, en la plataforma TEC Digital. Finalmente, en el módulo cinco cada educador debe sugerir rúbricas y materiales para cumplir con los objetivos de su curso. En la imagen 1 , se explican gráficamente los distintos módulos presentados, así como la duración en semanas, la descripción de cada módulo y el resultado esperado a partir de esta formación.

\section{RESULTADOS DEL ESTUDIO Y ANÁLISIS}

Cada actividad de este proceso de formación estuvo asociada a una estrategia de aprendizaje-evaluación. Se utilizaron herramientas tecnológicas diversas, para que desde su propia experiencia, cada profesor pudiera valorar su uso en el curso que dará en el futuro. Como se indica en la imagen 1, cada módulo de capacitación produjo resultados parciales que contribuyeron a cumplir el objetivo principal de la propuesta en estudio.

Los resultados se exponen a continuación de acuerdo con los tipos de evaluación diagnóstica, procesual y final aplicadas durante el desarrollo de los diferentes módulos.

\section{A. Evaluación diagnóstica}

El diagnóstico se realizó en diferentes fases. La primera fue valorar la necesidad de ofrecer una formación virtual para docentes que deseen enseñar español como segunda lengua a través de la modalidad en línea. La segunda fase se enfocó en determinar qué formación docente es necesaria e indispensable para futuros profesores virtuales. Finalmente, la tercera fase fue determinar el perfil del participante que matricula los cursos.

La primera fase del diagnóstico permitió determinar que la oferta de cursos de formación docente en la enseñanza del español como segunda lengua es un requerimiento esperado por potenciales participantes. Muchos de ellos se desplazan desde lugares alejados a los centros de formación presencial y esto 


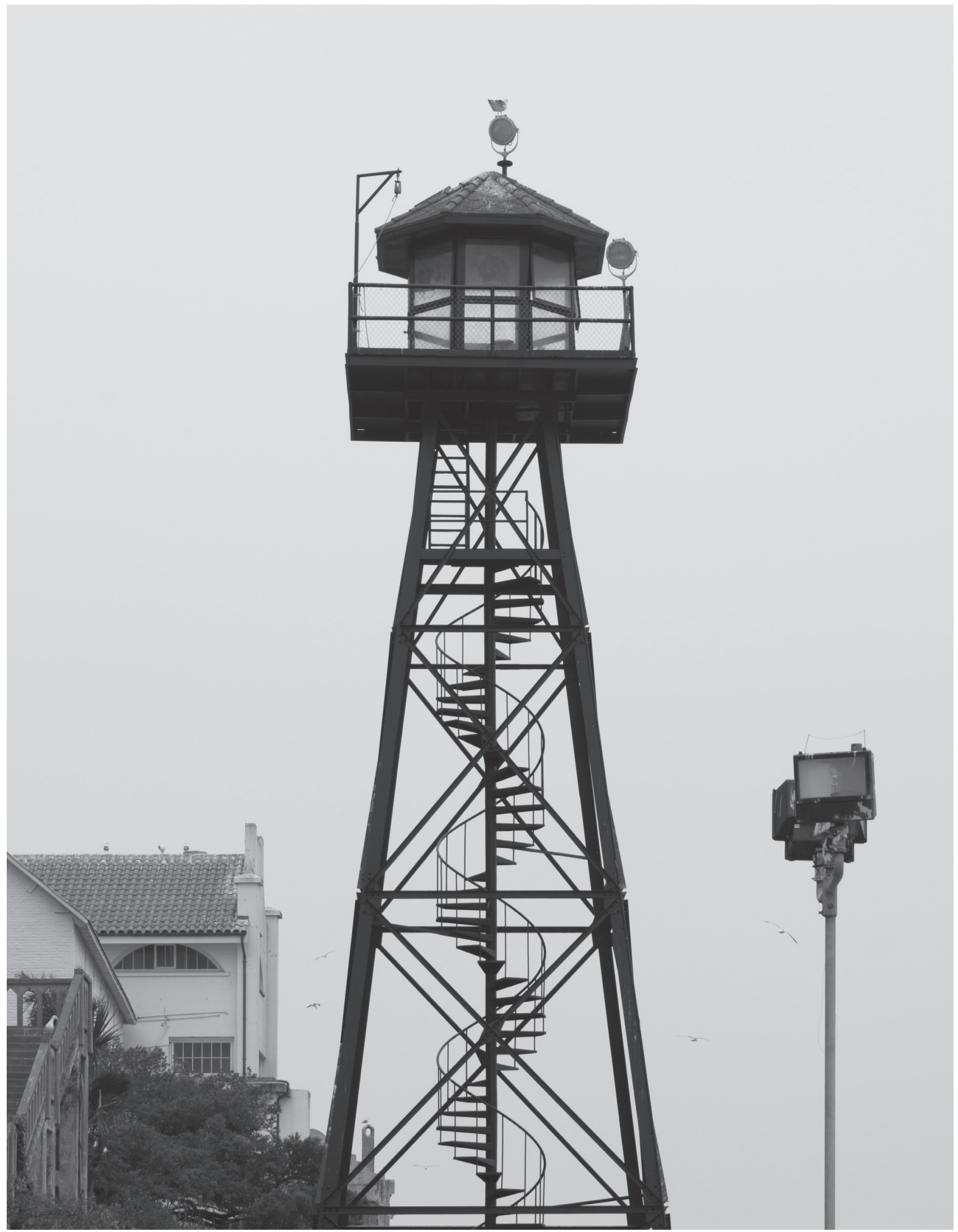

Fotografía de Leonardo Corrales N. 
tiene implicaciones de tiempo y costo. La opción de estudio virtual permitiría llegar a más personas en distintos lugares del país y fuera de sus fronteras.

De un total de 20 centros encuestados, por cada diez profesores de español como segunda lengua, ocho desean capacitarse en esta área debido a dos razones primordiales: una, no han recibido formación docente para crear cursos en línea, a pesar de tener un mínimo de cinco años de experiencia en el área. Y dos, porque consideran que la actualización es necesaria para conocer más herramientas tecnológicas, que les permitan planear una clase más dinámica e interactiva, de acuerdo con las exigencias de nuestro siglo.

Es importante señalar que ante la necesidad de formación docente con la cual obtener una certificación para enseñar español "online" se requiere de programas como este que preparen a los educadores en la programación de su propia clase virtual. Con ello, la experiencia vivida como alumnos, les permitió entender en ese rol, cuáles puedan ser las inquietudes de sus futuros educandos cuando se matriculen en sus cursos.

Por otra parte, el diagnóstico en su segunda fase generó un plan de formación para profesores con el objetivo de dar capacitación en el diseño de cursos virtuales, como se aprecia en la imagen 1. Cada módulo cumple un objetivo y un proceso de formación que contribuye a insertar a los participantes en el mundo de la educación virtual a partir de su propia vivencia y a desarrollar productos para sus clases en línea.

Para completar la evaluación diagnóstica las investigadoras determinaron el perfil del docente participante en términos tecnológicos, profesionales y personales. Esto permitió seleccionar cinco docentes del PT EESL como candidatos a participar en el proceso de formación. Cabe aclarar que todos son profesores en modalidad presencial, con afinidad y gusto tanto por la tecnología como por la enseñanza de un idioma. Ninguno de ellos tenía experiencia previa en recibir cursos $100 \%$ virtuales en un programa completo de formación. El título mínimo exigido para llevar un programa virtual es un bachillerato universitario ${ }^{4}$ en enseñanza de un idioma.

$4 \quad$ Un bachillerato universitario equivale a cuatro años de formación académica superior.
Este último aspecto obedece a que la experiencia previa como alumno en una universidad le permite ubicarse más fácilmente en un programa académico de nivel superior.

\section{B. Evaluación procesual}

Cuatro de los cinco participantes concluyeron exitosamente el proceso de formación, el cual permitió evaluar y realimentar el trabajo realizado por cada participante. El uso de diversas estrategias de aprendizaje y de evaluación permitió a los participantes generar experiencia y valorar su incorporación en el diseño instruccional de cada curso que tenían a cargo. De la misma forma, el hecho de utilizar distintas herramientas tecnológicas como facilidades de Facebook, wikis, foros, chat, videoconferencia, Google Drive y otras les permitió a los participantes evaluar su uso potencial para poderlas incorporar en sus clases. La persona que no concluyó fue por motivos personales que le impidieron continuar con el curso.

Parte de los resultados procesuales es la realimentación al plan de formación propuesto, realizada por parte de las investigadoras. Esto se obtuvo de la evaluación que los participantes hicieron de la labor de las investigadoras en aspectos relacionados con el desempeño docente, durante el proceso de formación en términos de: conocimiento, comunicación, disponibilidad para la atención de consultas y uso de la plataforma tecnológica. Las investigadoras obtuvieron un promedio de 95 a 100 en los diferentes aspectos. La única observación presentada fue la cantidad de material y actividades didácticas en cada uno de los módulos lo cual generó estrés en algunos casos.

\section{Evaluación final}

Al finalizar los cinco módulos, los productos generados por los participantes fueron:

1. El diseño instruccional que contempla objetivos, contenidos, estrategias de enseñanza y aprendizaje para ambientes virtuales, así como herramientas tecnológicas diversas y la especificación de sus respectivos recursos educativos.

2. Las pautas o consignas que "contienen indicaciones explícitas, taxativas sobre tareas o actividades que el docente solicita o sugiere al alumno." (Asinsten, 2007, p. 74). 
3. Material didáctico que incluye infografías, vídeos, audios, imágenes y textos, así como documentación para los respectivos cursos.

4. Al menos una rúbrica de evaluación por cada curso, que fueran compartidas entre los participantes.

\section{CONCLUSIONES}

A. La propuesta de un plan de formación docente para la creación de cursos virtuales, permitió:

1. El diseño de seis módulos (Introductorio más otros cinco) para la formación de docentes en la enseñanza del español como segunda lengua, cada uno con su respectivo DI, configurado en la plataforma TEC Digital. Para ello, fue necesaria la selección de criterios pedagógicos. De todos los analizados, el destacado por diversos autores, es el diseño instruccional, pues constituye la "columna vertebral" (Rodríguez, Espinoza y Moreira, 2014) de un curso, independientemente de la modalidad, pero en el caso de cursos virtuales es aún más relevante (Arshavskiy, 2014; Belloch, 2013; FAO, 2011; Gil, 2012).

2. Contribuir con lo indicado en el Plan Estratégico del TEC en relación con la incorporación de las TIC (ITCR, 2012) y también con lo estipulado, a nivel nacional, por CONARE (2011).

3. Tal y como lo indica Jiménez (2013), el profesor asume un papel de usuario y difusor de las tecnologías en el contexto educativo y el plan de formación docente contribuye a dar a conocer el uso y aplicación de las TIC en ambientes de educación superior.

4. Un programa caracterizado por su autonomía, motivación, interacción y comunicación periódica para los participantes. El resultado es un reflejo de lo que los docentes deberán hacer en el futuro cuando diseñen y ofrezcan sus cursos.

5. El plan de formación de los docentes es un factor crítico para la incorporación de las TIC en la academia y es necesario contar con un programa de formación del uso de las TIC porque: "La tecnología no enseña por sí misma. Su aplicabilidad se basa únicamente en la experiencia humana. La capacitación proporciona capacidad, habilidades y conocimientos que impulsarán el aprendizaje electrónico como una oferta educativa." (Odunaike, Olugbara, y Ojo, 2013, p. 4).

B. Los productos generados a partir de la implementación del plan de formación generaron:

1. A pesar de que ningún profesor participante había tenido la experiencia en recibir cursos $100 \%$ virtuales, en este estudio se logró que los participantes desarrollaran: la primera versión de cuatro cursos en modalidad virtual con su diseño instruccional, las pautas didácticas para los estudiantes sobre cada módulo, así como las rúbricas de evaluación y los materiales didácticos, acordes con los objetivos propuestos. Siempre hubo un trabajo responsable, comprometedor y proactivo en el proceso.

Lo anterior permite realizar una reflexión sobre el perfil de quien ingresa al programa virtual. En un principio se pensaba en que debía tener una experiencia previa en este tipo de cursos, de ahí que el plan piloto sirviera para probar si esto era necesario. Como se puede observar, el resultado fue satisfactorio. Por lo tanto, para cursos futuros, no será necesario que la persona haya pasado por cursos en línea. Lo importante es que haya tenido un desempeño en la formación de estudiantes, específicamente en la enseñanza de un idioma.

Los cuatro cursos propuestos fueron: Gramática aplicada, Niveles comunes de referencia para las lenguas, Planeamiento didáctico y Medios digitales como herramientas didáctica. Desde el punto de vista de la enseñanza del español como segunda lengua, se incorporó a cada curso la teoría del MCER (2002) y la del Instituto Cervantes (2006), según el Plan Curricular publicado. También se realizó la propuesta de actividades sincrónicas y asincrónicas, se incorporaron el $50 \%$ de las herramientas dadas por 
Formación de docentes para la creación de cursos virtuales en la enseñanza del español como segunda lengua

el mismo curso de formación docente y se seleccionaron documentos digitales actualizados y con información relevante sobre los temas asignados.

Sobre el capítulo Las tareas y el papel en la enseñanza de la lengua, del MCER (2002), se incorporó la teoría específica sobre la importancia de este tema para el educando. "Las tareas pueden tener un carácter enormemente variado y pueden comprender actividades de la lengua en mayor o menor medida, como, por ejemplo: (...) actividades basadas en destrezas, resolución de problemas (rompecabezas, crucigramas), transacciones habituales, (...) participación en un debate o foro virtual, planificación de una acción, lectura y respuesta a un mensa$\mathrm{je}^{\prime \prime}$ (MCER, 2002, p. 155). Todas estas fueron consideradas en cada uno de los cursos planeados.

2. La primera versión del perfil de los potenciales estudiantes del programa en su modalidad virtual, contempla factores personales, tecnológicos y de autodeterminación (Gil, 2012). La importancia de este producto lo describe Gil $(2012$, p. 3) en el sentido de "Tener presente las características de los estudiantes facilita el diseño e implementación de un programa educativo, ya que se 'diseña a la medida' y de acuerdo con las necesidades que se van a cumplir.".

Cada curso de la propuesta presenta las siguientes características:

a. Aprovecha las TIC en función de los participantes, y específicamente en su aprendizaje, según lo recomiendan Salinas, Pérez y De Benito (2008).

b. Los profesores, basados en su experiencia práctica, eligieron las herramientas tecnológicas que utilizarán en sus cursos. En este sentido, los participantes incluyeron en el diseño instruccional, de acuerdo con el criterio desarrollado, estrategias de aprendizaje individuales y colaborativas, que a su vez se implementan con herramientas digitales como wikis, foros, blogs o videoconferencia, entre otros.

c. El proceso de virtualización consideró y aplicó criterios pedagógicos y tecnológicos que, de acuerdo con expertos, son factores críticos para garantizar la calidad de la formación virtual, tal es el caso del diseño instruccional como mapa de ruta para la enseñanza y el aprendizaje, el uso de rúbricas de evaluación, actividades de aprendizaje y evaluación enfocadas en el estudiante.

Además, contar con materiales educativos que cumplan criterios de calidad y planificados para contribuir con el aprendizaje, estrategias para la comunicación periódica y activa entre profesores y estudiantes (Alfonzo, 2011; Área, San Nicolás y Fariña, 2010; Cabero, 2006; Clark y James, 2012; FAO, 2011; González y Farnóz, 2009; Imbernón, Silva y Guzmán, 2011; Ortega, 2007; Ortega y García, 2002; Sae-Khow, 2014; Salinas, Pérez y De Benito, 2008; Sarsa y Soler, 2012).

3. A partir de la interacción, se creó un inventario de herramientas digitales que pueden ser utilizadas por los mismos profesores. A lo largo del programa, se fueron probando diferentes recursos tecnológicos con el fin de que los participantes analizaran si estos les podían servir a futuro, y de qué manera los podían enlazar con los contenidos de los cursos asignados. Se utilizó para esto el Google Drive, con el objetivo de que los interesados, pudieran editar un solo documento, y construir entre todos, las sugerencias de las actividades curriculares para cada una de las herramientas sugeridas.

\section{REFERENCIAS BIBLIOGRÁFICAS}

Alfonzo, A. (2011). Caracterización de algunas dimensiones de la interacción didáctica en la modalidad de educación a distancia: Caso: Universidad Nacional Abierta de Venezuela. (Disertación Doctoral) Universidad Nacional de Educación a Distancia, Venezuela. 
Área, M., San Nicolás, M. B. y Fariña, E. (2010). Buenas prácticas de aulas virtuales en la docencia universitaria semipresencial. Teoría de la Educación. Educación y Cultura en la Sociedad de la Información, 11 (1), 7-31.

Arshavskiy, M. (2014). Diseño Instruccional para Aprendizaje en línea: Guía esencial para la creación de cursos exitosos de educación en Línea. Estados Unidos: Your ELearning World.

Asinsten, J. (2007). Producción de contenidos para la educación virtual. Biblioteca digital Virtual Educa. Recuperado de http://reposital.cuaed.unam. mx:8080/jspui/handle/123456789/3447.

Belloch, C. (2013) Diseño Instruccional. Universidad de Valencia. Recuperado de http://www. uv.es/bellochc/pedagogia/EVA4.pdf

Cabero-Almenara, J. (2006). Bases pedagógicas del e-learning. RUSC. Universities and Knowledge Society Journal, 3 (1), 1.

Clark, I., y James, P. (2012, October). Blended learning: An approach to delivering science courses on-line. In Proceedings of The Australian Conference on Science and Mathematics Education (formerly UniServeScience Conference) (Vol. 11).

CONARE. (2011). Plan Nacional de Educación Superior Universitaria Estatal 2011-2015. San José Costa Rica.

Consejo de Europa. (Junio, 2002) Marco Común Europeo de Referencia para las lenguas: aprendizaje, enseñanza y evaluación. Madrid: España. En: http://cvc.cervantes.es/ensenanza/biblioteca_ele/ marco/cvc_mer.pdf

Ejarque, E., Buendía, F. y Hervás, A. (2008). Aplicación de un modelo de calidad para evaluar experiencias e-learning en el Espacio Europeo Universitario. Educar (41), 11-28.

Fernández-Robles, B., y Cabero, J. (2016). Percepciones de teleformadores del Instituto de Formación y Estudios Sociales de Sevilla sobre la teleformación. Innoeduca. International Journal of Technology and Educational Innovation, 2 (1), 4-12.

Fallas, I. (2005). El uso de rúbricas para la evaluación en los cursos on line. Ponencia presentada en la Conferencia Internacional de Educación a
Distancia, en San Juan Puerto Rico (agosto, 2005). Descargado de http://www.uned.ac.cr/ece/images/documents/documents2010/Articulo_de_Rubricas.pdf

FAO. (2011). E-learning methodologies. A guide for designing and developing e-learning courses. Rome, Italy.

García-Aretio, L. (2009). Concepción y tendencias de la educación a distancia en América Latina. Madrid, España: Centro de Altos Estudios Universitarios de la OEl.

Gatica-Lara, F. y Uribarren-Berrueta, T. (2013). ¿Cómo elaborar una rúbrica? Investigación en Educación Médica, 2 (5), 61-65.

Gil, M. (2004) Modelo de Diseño instruccional para programas educativos a distancia. Perfiles Educativos, (26), 104, 93-114.

Gil, M. (2012). Modelo didáctico para la formación de equipos multidisciplinarios para la implementación de programas educativos en línea. Virtual Educa. Recuperado de http://reposital.cuaed. unam.mx:8080/jspui/handle/123456789/3447.

González, A. y Farnóz, J, (2009). Usabilidad y accesibilidad para un e-learning inclusivo. Revista Educación Inclusiva (2), 1.

Henríquez, G., Veracoechea, B., Papale, J. F. y Berrios, A. T. (2015). Modelo de capacitación docente para entornos virtuales de aprendizaje. Caso Decanato Ciencias de la Salud de la UCLA. Revista Iberoamericana de Educación a Distancia, 18 (1), 67.

Imbernón, F., Silva, P. y Guzmán, C. (2011). Competencias en los procesos de enseñanza-aprendizaje virtual y semipresencial. Comunicar, (18). 36, 107-114. doi:10.3916/C36-2011-03-01

Instituto Tecnológico de Costa Rica [ITCR]. (2012) Plan Estratégico 2011-2015. Cartago.

Instituto Cervantes. (2006). Plan Curricular del Instituto Cervantes. Niveles de Referencia para el español. Madrid: Editorial Biblioteca Nueva, S.L.

Jiménez, L. (2013). Concepciones sobre la evaluación en los cursos con modalidad b-learning. Ibagué: Universidad del Tolima. Recuperado de http://repository.ut.edu.co/handle/001/1037. 
MCER. (2002). Marco Común Europeo de Referencia para las Lenguas: Aprendizaje, Enseñanza, Evaluación. Recuperado de http://cvc.cervantes. es/obref/marco.

Nieto, H. I. (2013). Propuestas para un modelo pedagógico virtual para EAD. Signos Universitarios, 31 (48), 63-85.

Odetti, V. (2013). El diseño de materiales didácticos hipermediales para los niveles medio y superior: experiencias incipientes en Argentina. Recuperado de: http://www.pent.org.ar/institucional/publicaciones/diseno-materiales-didacticos-hipermediales-para-niveles-medio-superior-e.

Odunaike, S. A., Olugbara, O. O. y Ojo, S. O. (2013). E-learning Implementation Critical Success Factors (Vol. I). Presentado en International MultiConference of Engineers and Computer Scientists 2013, Hong Kong. Recuperado de: http://www.iaeng.org/publication/IMECS2013/ IMECS2013_pp560-565.pdf.

Ortega, J. (2007). Nuevas tecnologías para la educación en la era digital. España: Editorial Pirámide.

Ortega, J., y García, A. (2002). Creando cultura evaluadora de la calidad de los materiales didácticos usados en la formación ONLINE. En: Alonso, C., Gallego, D. (Eds.), (2002) Tecnologías de la información y la comunicación para el aprendizaje. Tomo I. Madrid: UNED.

Pastor, S. (2005). Español como segunda lengua: difusión, aprendizaje y formación. Hispanogalia, Consejería de Educación de la Embajada de España en Francia, 53-167.

Pernalete, D., Cánchica, M. y Coello, Y. (2010). Estándares y Modelo de Calidad asociado a los ambientes de enseñanza-aprendizaje para E-learning. Coro, Falcón, Venezuela: Universidad Nacional Experimental Francisco de Miranda.

Rico, D. (2012) Modelo de evaluación y fortalecimiento de la educación virtual desde el enfoque de los procesos de enseñanza-aprendizaje. Tesis para optar por el título de Magíster en Gestión de Informática y Telecomunicaciones. Santiago de Cali, Colombia. Recuperado de: http:// repository.icesi.edu.co/biblioteca_digital/bitstream/10906/71185/1/modelo_evaluacion_fortalecimiento.pdf.

Rodríguez, O. (2012). La satisfacción del usuario en la enseñanza virtual: el caso del sistema universitario andaluz. Recuperado de: http://0-hera. ugr.es.adrastea.ugr.es /tesisugr/21613084.pdf.

Rodríguez, N., Espinoza, J. y Moreira, T. E. (2014). Evaluación de los aprendizajes en ambientes virtuales o bimodales: Un análisis multivariado. Instituto Tecnológico de Costa Rica: Costa Rica.

Sae-Khow, J. (2014). Developing of Indicators of an E-Learning Benchmarking ModelforHigherEducationInstitutions. Turkish Online Journal of EducationalTechnology-TOJET, (13), 2, 35-43.

Salinas, J., Pérez, A. y De Benito, B. (2008). Metodologías centradas en el alumno para el aprendizaje en red. Madrid, España: Editorial Síntesis.

Sarsa, J. y Soler, R. (2012). E-learning quality: Relations and perceptions. International Journal of Information and Communication Technology Education (IJICTE), 8 (2), 46-60.

Savic, S., Stankovic, M. y Janackovic, G. (2012). Hybrid model for e-learning quality evaluation. International Journal of Emerging Technologies in Learning, (iJET), 7.

Solano, O. (2010). Las posibilidades que brinda el Aprendizaje combinado (modalidad presencial y no presencial apoyado por el uso de Internet), para el desarrollo de las destrezas de comunicación escrita. Proyectos Investigación Costa Rica. Recuperado de: www.tec.ac.cr/sitios/Vicerrectoria/vie/paginas/proyectos investigacion.

Wang, L. (2007). Sociocultural learning theories and information literacy teaching activities in higher education. Reference \&User Services Quarterly, 47 (2), 149-158.

Videla, J. (s.f.). Evaluación. Pedagogía Básica, Didáctica y Evaluación de las Ciencias Integradas, Centro Puente Alto, Universidad Arturo Prat. Arrayán: Chile. Recuperado de: https://juanvidela. files.wordpress.com/2010/03/pedagogia-basicaevaluacion-unap_ciencias-integradas.pdf. 